\title{
On the relationship between structure and reaction rate in olefin ring-closing metathesis $\dagger$
}

\author{
Ian W. Ashworth, ${ }^{a}$ Davide Carboni, ${ }^{b}$ Ian H. Hillier, ${ }^{c}$ David J. Nelson, ${ }^{b}$ Jonathan M. Percy, ${ }^{* b}$ \\ Giuseppe Rinaudo ${ }^{b}$ and Mark A. Vincent ${ }^{c}$
}

Received 8th July 2010, Accepted 17th August 2010

DOI: $10.1039 / \mathrm{cocc02440f}$

In the RCM reactions of a series of simple $\alpha, \omega$-dienes, the relative order of reactivity has been unambiguously determined showing that cyclohexene forms faster than cyclopentene or cycloheptene. 1,5-Hexadiene inhibits the RCM of 1,7-octadiene; 1,5-hexadiene cannot progress to the RCM product (cyclobutene) but forms an unexpectedly stable cyclic $\eta^{2}$-complex.

Alkene metathesis has become a vital part of the synthetic repertoire, and has been applied in many syntheses of complex natural products. Much of what we know about the relationship between diene structure and reactivity has been inferred from reaction yields from the huge body of results that has accumulated in the area of target synthesis; quantitative studies that relate structure and reactivity are very rare. ${ }^{1,2}$

Arguments based on kinetic versus thermodynamic control have been advanced by Schmidt and $\mathrm{Nave}^{3}$ to explain reaction outcomes from systems that can form 5- or 6-membered rings competitively. For example, Scheme 1 shows the completely selective double RCM of $\mathbf{1}$ to $\mathbf{2}$ when exposed to the first generation Grubbs pre-catalyst $\mathbf{3}$. Because $\mathbf{3}$ is known to react slowly with disubstituted alkenes (and particularly those with an allylic oxygen function), it seems extremely unlikely that 2 could be a thermodynamic product. Substrate $\mathbf{4}$ with a free hydroxyl group affords dihydrofuran $\mathbf{5}$ and dihydropyran $\mathbf{6}$ in equal proportions and an hydroxyl group directing effect is
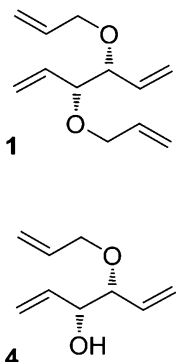
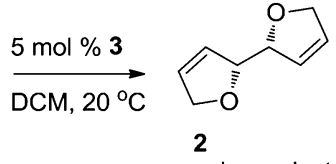

sole product

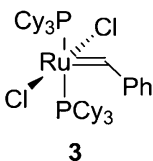

3

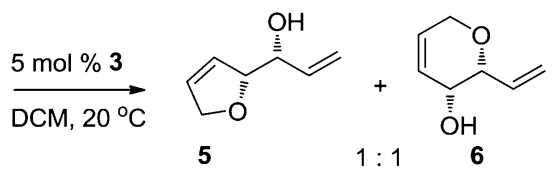

Scheme 1 invoked to explain the appearance of the 6-membered ring product. It is also interesting that various attempts to populate 6-membered ring species as thermodynamic products by raising reaction temperatures and applying an overpressure of ethene failed to deliver clean results. The presence of functional groups on the substrates used for target synthesis inevitably complicates the analysis of reaction outcomes.

We were therefore interested to discover if there were significant rate differences in RCMs of simple $\alpha, \omega$-dienes in solution; rigorous characterisation of the behaviour of the parent diene systems would then enable the assignment of kinetic products to be made more confidently.

A recent mass spectrometric study by Metzger and co-workers ${ }^{4}$ identified ions on the cyclisation pathways of simple $\alpha, \omega$-dienes 7a-d catalysed by Grubbs' first generation pre-catalyst 3 (Scheme 2). Collisional energies were used to estimate the rates at which the $\eta^{2}$-complexes $\mathbf{8 a - d}$ progressed onward through the cycle. Metzger reported that the most reactive system derived from 1,7-octadiene $7 \mathbf{c}(n=2)$, which goes on to form cyclohexene; the $\eta^{2}$-complex $8 \mathbf{c}$ progressed more rapidly than those from 1,6-heptadiene $7 \mathbf{b}(n=1)$ and 1,8-nonadiene $7 \mathbf{d}(n=3)$, which were very similar. This is contrary to what appears to be a generally held belief that 5 -membered rings are the fastest to form by RCM. ${ }^{3,4}$

We have investigated the cyclisation rates of simple $\alpha, \omega$-dienes in solution using the Grubbs' second generation pre-catalyst 9 (Scheme 2) which is reactive enough to permit the cycloalkenes to equilibrate fully. ${ }^{5}$ NMR spectroscopy was the method of choice because it would allow concentrations to be measured without the introduction of artefacts arising from sample concentration or loss by evaporation. In the first instance, a mixture of $7 \mathbf{b}(3.3 \mathrm{mM}), 7 \mathbf{c}(3.4 \mathrm{mM})$ and $7 \mathbf{d}$ (3.5 mM) in $\mathrm{CDCl}_{3}$ containing 1,3,5-trimethoxybenzene as the internal standard, ${ }^{6}$ was prepared and exposed to pre-catalyst 9 $\left(1 \mathrm{~mol} \%\right.$ ) at $25{ }^{\circ} \mathrm{C}$ in the magnet of a $600 \mathrm{MHz}$ spectrometer (see the ESI $\dagger$ for full details). We were careful to determine

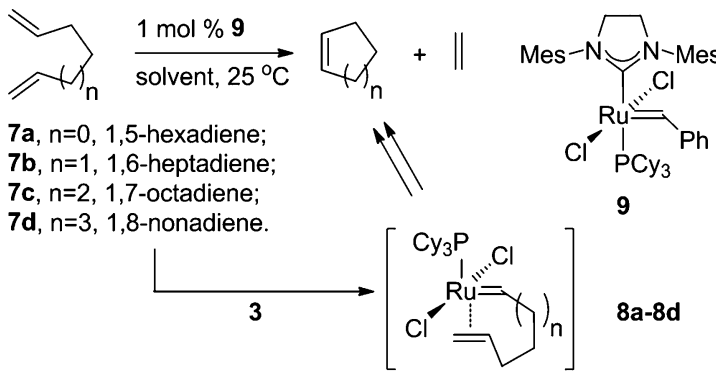

Scheme 2

${ }^{a}$ AstraZeneca Global Research and Development,

Silk Road Business Park, Charter Way, Macclesfield, SK10 2NA, UK

${ }^{b}$ WestCHEM Department of Pure and Applied Chemistry,

Thomas Graham Building, 295 Cathedral Street, Glasgow G1 1XL,

UK.E-mail: jonathan.percy@strath.ac.uk;

Fax: + 44 (0)141 548 4822; Tel: + 44 (0)141 5484398

${ }^{c}$ School of Chemistry, University of Manchester, Oxford Road, Manchester M13 9PL, UK

$\dagger$ Electronic supplementary information (ESI) available: $T_{1}$ values for dienes and cycloalkenes, typical NMR spectra of reaction solutions, concentration/time data and plots, Cartesian coordinates, and energies of 10a-c, 11a-c, 12b, 12c, 13b and 13c. See DOI: $10.1039 / \mathrm{c} 0 \mathrm{cc} 02440 \mathrm{f}$ 


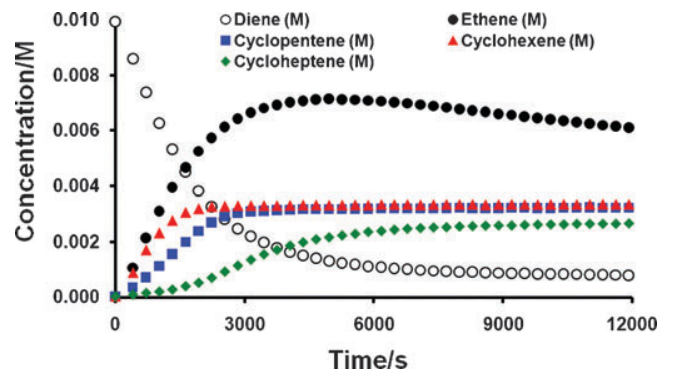

Fig. 1 Competition experiment in chloroform showing product formation.

$T_{1}$ 's for all the reaction components, and set $D_{1}$ to $5 T_{1}$ to ensure accurate integration. Dichloromethane, rather than chloroform, is the default solvent for RCM in the synthetic literature; we elected to work in $d$-chloroform because it was more economical, but selected experiments were also run in $d_{2}$-dichloromethane.

The dispersion between key signals was excellent and we were able to collect a full concentration/time profile for each cycloalkene as the reactions proceeded to their end points (Fig. 1). The identity of the most reactive diene was beyond dispute; cyclohexene formed most rapidly from the reaction, in agreement with Metzger's earlier inference. Cyclopentene formed less rapidly, and cycloheptene was the slowest of the three. We carried out a series of single substrate experiments (each in duplicate) and were able to reproduce the reactivity order obtained from the three-substrate experiment, indicating that the profiles do not represent an artefact of a competition process. This finding clearly contradicts the assertion that 5-membered rings in general form fastest by RCM.

It was also possible to measure the solution concentration of ethene, which reaches $90-95 \%$ of its maximum value before slow egress begins. ${ }^{7}$ The second generation pre-catalyst 9 was also present at the end of the reaction (from the ${ }^{1} \mathrm{H}$ NMR spectrum), so these mixtures may represent equilibrium compositions because all the reaction components are present. In both dichloromethane and chloroform, $7 \mathbf{b}$ and $7 \mathbf{c}$ were converted completely within experimental error to their cycloalkene products (all the reactions were slightly faster in dichloromethane), whereas the reaction of 1,8-nonadiene 7d forms small quantities ( $c a .5 \%$ ) of oligomeric material in addition to cycloheptene in either solvent. ${ }^{8}$ There was a minor difference in half-life in the two solvents for all the dienes, with the chloroform reactions typically slightly slower. $\ddagger$

The behaviour of 1,5-hexadiene 7a, was also examined; Metzger and coworkers found that the initial $\eta^{2}$-complex derived from this diene and pre-catalyst 3 was much more stable than expected. Other authors have suggested that chelate complexes containing a hexadienyl motif are stabilised; 7a is known to undergo ADMET polymerisation more slowly than 1,9-decadiene, for example. ${ }^{9-11}$

We therefore examined the behaviour of this shorter diene under the conditions used to cyclise $\mathbf{7 b}-\mathbf{7 d}$ and found that $\mathbf{7 a}$ was unreactive at $10 \mathrm{mM}(1 \mathrm{~mol} \%$ 9), with no change in the ${ }^{1} \mathrm{H}$ NMR spectrum; even at $250 \mathrm{mM}$, conversion was very slow, reaching only $20 \%$ after 8 hours. In two further experiments, the first contained an equimolar $(4.5 \mathrm{mM}$ in each) mixture of $7 \mathbf{b}$ and $7 \mathrm{c}(1.1 \mathrm{~mol} \% \mathbf{9}$ based on total diene concentration),

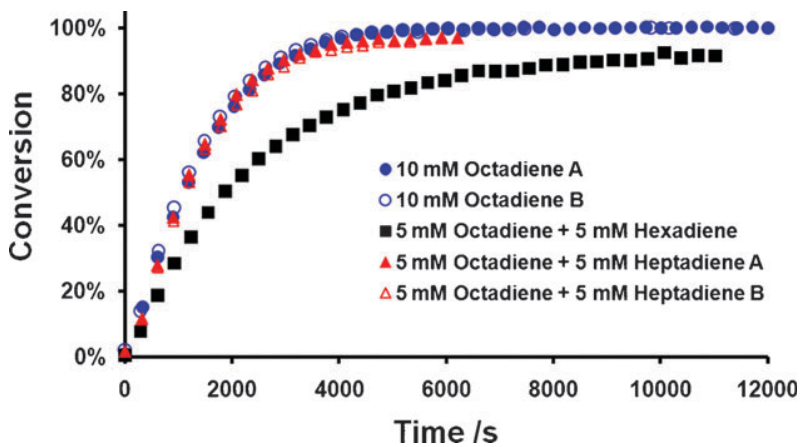

Fig. 2 Inhibition of cyclohexene formation by hexadiene.

while the second mixed 7a and 7c $(5 \mathrm{mM}$ in $7 \mathbf{c}, 5.7 \mathrm{mM}$ in $7 \mathbf{a}$ with $0.93 \mathrm{~mol} \% 9$ ). While the conversion profile of cyclohexene formation in the $\mathbf{7 b} / \mathbf{7} \mathbf{c}$ mixture was almost identical to that from the experiment containing $7 \mathrm{c}$ alone $(10 \mathrm{mM}, 1 \mathrm{~mol} \% 9)$, cyclohexene formed significantly more slowly and reached lower conversion when $7 \mathbf{a}$ was present in the reaction mixture (Fig. 2).

Electronic structure calculations at the M06-L/B2/CPCM// M06-L/B2 level were used to probe further. We have previously shown that the M06-L functional is appropriate for describing these systems. ${ }^{12}$ The basis used, B2, consisted of the SDD ECP and corresponding basis set on $\mathrm{Ru},{ }^{13}$ with an $f$-function of exponent 0.5780 , and with a $6-311 \mathrm{G}^{* *}$ basis on all other atoms. This calculation gave quite different geometries for the two initial $\eta^{2}$-complexes (Fig. 3). The $\eta^{2}$-complex 11c (Fig. 3b) lies $6.6 \mathrm{kcal} \mathrm{mol}^{-1}$ below the starting $14 \mathrm{e}$ alkylidene complex. In this $\eta^{2}$-complex the $\pi$ orbitals of the $\mathrm{C}=\mathrm{C}$ and $\mathrm{Ru}=\mathrm{C}$ double bonds point towards each other and are thus ready to form the metallocycle, whereas in the $\eta^{2}$-complex from 1,5-hexadiene (11a, Fig. 3a) the $\pi$ orbitals are at right angles, so this is not a reactive conformation. This complex lies $10.6 \mathrm{kcal} \mathrm{mol}^{-1}$ below the starting 14e alkylidene complex, and is noticeably more stable than 11c. This arrangement of alkene and alkylidene is very similar to the one found in an untethered methylidene/ethene complex. ${ }^{14}$ As shown in Fig. 4, $\eta^{2}$-complex 11a is competitive with the lowest point (metallocyclobutane $12 \mathrm{c}$ at $-10.2 \mathrm{kcal} \mathrm{mol}^{-1}$ ) on the energy surface for cyclohexene formation. These results confirm the origin of the effect observed by Metzger and begin to explain the very low cross metathetical reactivity of 7a. They also support the observation that $\mathbf{7 a}$ retards cyclohexene formation, presumably by sequestering active catalyst in a non-productive complex, ${ }^{15}$ competitively with productive binding to $7 \mathbf{c}$. The heptadiene metathesis begins with complex $\mathbf{1 1 b}$; while this is also a much more stable complex than 11c, it can progress and therefore does not sequester catalyst. Inspection of the next

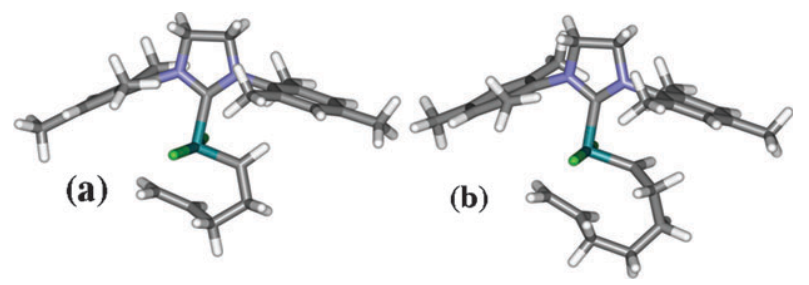

Fig. 3 Optimised geometries (M06-L/B2) for (a) 11a and (b) 11c. 

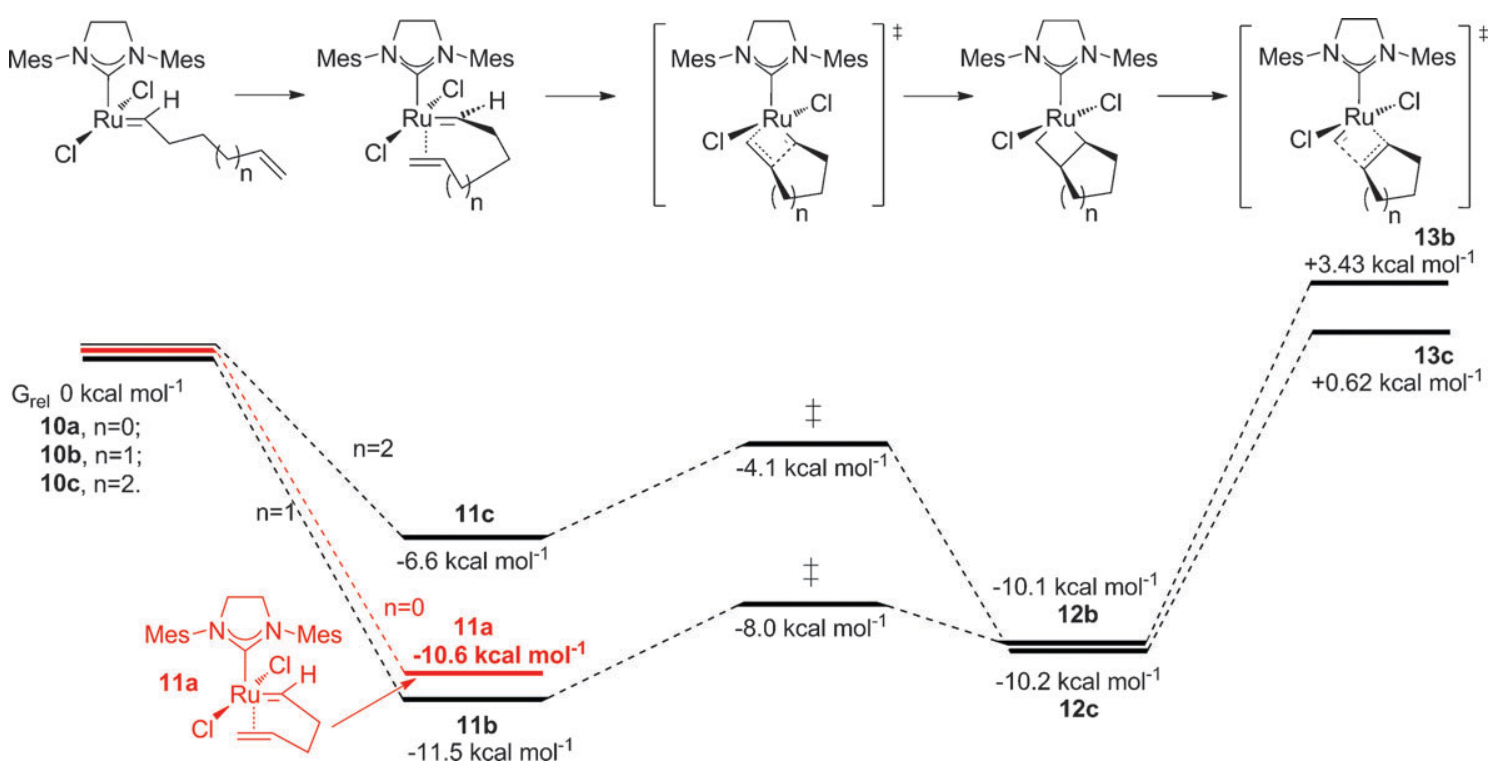

Fig. 4 Partial free energy profiles for metathesis of hexadiene 7a (red), and heptadiene 7b and octadiene 7c (black) with pre-catalyst 9.

part of the energy surface predicts that cyclohexene will form more quickly than cyclopentene if metallocyclobutane breakdown is rate-determining, consistent with the behaviour of the dienes in the NMR experiments.§

In conclusion, we have shown that the order of kinetic reactivity $(6>5>7)$ of simple $\alpha, \omega$-dienes in RCM is not the one assumed in the literature, or referred to in investigations of more complex systems; cyclohexene has been shown to form more rapidly than cyclopentene, with cycloheptene formation significantly slower. Further, the 1,5-hexadienyl motif is shown to be far from innocent in metathesis, with 1,5-hexadiene inhibiting the rapid cyclisation to cyclohexene. While cyclobutene does not form, care should be taken in synthetic planning lest the formation of stable $\eta^{2}$-complexes analogous to 11a impede RCMs involving complex substrates.

This work was supported by AstraZeneca (Industrial CASE Award to DJN), the EPSRC Initiative in Physical Organic Chemistry 2 (EP/G013160/1 and EP/G013020/1, fellowship to DC) and the University of Strathclyde (fellowship to GR).

\section{Notes and references}

$\ddagger$ Only the reaction of $\mathbf{7 c}$ in dichloromethane was clearly first order, so we cannot use first order rate constants to quantify the differences between the reactions at this stage. The minor difference in half-life observed for all the dienes suggests that $\mathrm{CDCl}_{3}$ is a useful solvent for the study of these reactions.

$\S$ Metallocyclobutane breakdown was identified as the highest energy barrier on the surface for cyclohexene formation in our earlier publication. ${ }^{12}$ However, catalyst formation from 9 and initial alkylidene transfer from $\mathbf{1 0}$ to $\mathbf{1 1}$ may be at least partially ratedetermining, ${ }^{16}$ making the identification of the rate determining step quite complex. A full analysis of the energy surfaces of these and higher dienes lies beyond the scope of this manuscript and will be reported elsewhere.

1 A. H. Hoveyda and A. R. Zhugralin, Nature, 2007, 450, 243.

2 Recently, Monfette and Fogg have emphasised the importance of thermodynamic control as a general determinant of RCM outcome. See: S. Monfette and D. E. Fogg, Chem. Rev., 2009, 109, 3783 .

3 B. Schmidt and S. Nave, Adv. Synth. Catal., 2007, 349, 215.

4 H. Y. Wang, W. L. Yim, T. Kluner and J. O. Metzger, Chem.-Eur. J., 2009, 15, 10948.

5 C. W. Lee and R. H. Grubbs, Org. Lett., 2000, 2, 2145.

6 C. S. Adjiman, A. J. Clarke, G. Cooper and P. C. Taylor, Chem. Commun., 2008, 2806.

7 For a recent measurement of ethene solubility, see: E. F. van der Eide, P. E. Romero and W. E. Piers, J. Am. Chem. Soc., 2008, 130, 4485. The roles of ethene and the alkylidene population will be discussed more fully elsewhere.

8 Kress has generated and characterised oligomeric species by ROMP of cycloheptene; J. Kress, J. Mol. Catal. A, 1995, $102,7$.

9 Z. Wu, A. D. Benedicto and R. H. Grubbs, Macromolecules, 1993, 26, 4975.

10 K. B. Wagener, K. Brzezinska, J. D. Anderson, T. R. Younkin, K. Steppe and W. DeBoer, Macromolecules, 1997, 30, 7363.

11 For an isolated $\eta^{2}$-complex containing a 1,5-hexadienyl motif, see: J. A. Tallarico, P. J. Bonitatebus and M. L. Snapper, J. Am. Chem. Soc., 1997, 119, 7157.

12 S. Pandian, I. H. Hillier, M. A. Vincent, N. A. Burton, I. W. Ashworth, D. J. Nelson, J. M. Percy and G. Rinaudo, Chem. Phys. Lett., 2009, 476, 37.

13 D. Andrae, U. Haeussermann, M. Dolg, H. Stoll and H. Preuss, Theor. Chim. Acta, 1990, 77, 123.

14 B. F. Straub, Angew. Chem., Int. Ed., 2005, 44, 5974.

15 I. C. Stewart, B. K. Keitz, K. M. Kuhn, R. M. Thomas and R. H. Grubbs, J. Am. Chem. Soc., 2010, 132, 8534.

16 E. L. Dias, S. T. Nguyen and R. H. Grubbs, J. Am. Chem. Soc., 1997, 119, 3887 